\title{
Relationship of sociodemographic conditions with the formation of body image in Brazilian adolescents
}

\section{Relação das condições sociodemográficas \\ com a formação da imagem corporal em adolescentes brasileiros}

\author{
Leticia Spricido BATISTA 1 (ID) 0000-0001-9525-3323 \\ Hélida Ventura Barbosa GONCALVES² (D) 0000-0003-2272-1768 \\ Daniel Henrique BANDONI ${ }^{\text {ID }}$ 0000-0003-1638-1437
}

A B S T R A C T

\section{Objective}

To investigate the associations between sociodemographic population conditions and adolescents' body image

\section{Methods}

This is a cross-sectional study using data from the Pesquisa Nacional de Saúde do Escolar (PeNSE, National School Health Survey), conducted in 2015. The objective was to analyze the relationship between students' sociodemographic conditions and body image attending public and private schools in Brazil capitals and the Federal District. For the statistical analysis, we conducted the Poisson regression models, generating adjusted prevalence ratios and their respective $95 \%$ $\mathrm{Cl}$. In the multiple models, sociodemographic variables were included, which were significantly associated with body image variables $(p<0.05)$ or which altered the prevalence ratios by at least $10 \%$.

\footnotetext{
1 Universidade Federal de São Paulo, Instituto de Saúde e Sociedade, Programa de Pós-Graduação em Alimentos, Nutrição e Saúde. Baixada Santista, SP, Brasil.

2 Universidade Federal de São Paulo, Instituto de Saúde e Sociedade, Programa Interdisciplinar em Ciências da Saúde. Baixada Santista, SP, Brasil.

${ }^{3}$ Universidade Federal de São Paulo, Instituto de Saúde e Sociedade, Departamento de Saúde. R. Silva Jardim, n. 136. Vila Matias, 11015-020, Santos, SP, Brasil. Correspondence to: Email: <bandoni@unifesp.br>.

Article elaborated from the dissertation by LS BATISTA, entitled "Imagem Corporal E Suas Dimensões Em Relação Às Condições Sociodemográficas E Práticas Alimentares De Adolescentes Brasileiros: Resultados Da Pesquisa Nacional De Saúde Do Escolar 2015". Universidade Federal de São Paulo, 2020.
}

How to cite thisarticle

Batista LS, Gonçalves HVB, Bandoni DH. Relationship of sociodemographic conditions with the formation of body image in Brazilian adolescents. Rev Nutr. 2021;34:e210056. https://doi.org/10.1590/1678-9865202134e210056 


\section{Results}

The research involved 10,926 adolescentes The analyses showed that the older the adolescents, the greater their dissatisfaction with their body. As for the school administrative status, students from private schools were more unhappy with their image (Girls: 33\% and Boys: 17.6\%) and, they tend to have more attitudes to lose weight (Girls: $42.8 \%$ and Boys: $28 \%$ ). The mother's level of education causes daughters to be more dissatisfied with their bodies (33.3\%) and sons showing more attitudes of wanting to lose weight (26.6\%).

\section{Conclusion}

Sociodemographic conditions are directly and indirectly associated with the adolescents' body image. Negative deviations in body image can result in binge eating and social disorders hindering the development of these pubescent children. The consequences of these actions become a risk to health and psychological well-being, strongly supporting the initiative of programs aimed at issues related to body image in the school setting.

Keywords: Body Image. Physical Appearance. Public Health. Teenager.

\section{RE S U M O}

\section{Objetivo}

Investigar as associações das condições sociodemográficas com a imagem corporal dos adolescentes no âmbito populacional.

\section{Métodos}

Trata-se de um estudo transversal, o qual utilizou dados da Pesquisa Nacional de Saúde do Escolar (PeNSE) realizada em 2015. Para analisar as relações das condições sociodemográficas com a imagem corporal, foram avaliados alunos de escolas públicas e privadas das capitais brasileiras e no Distrito Federal. As análises ocorreram por meio de modelo de regressão de Poisson, gerando razões de prevalências ajustadas e seus respectivos IC95\%. Nos modelos múltiplos, foram incluídas as variáveis sociodemográficas que estivessem significativamente associadas às variáveis de imagem corporal $(p<0,05)$ ou que alterassem as razões de prevalência em pelo menos $10 \%$.

\section{Resultados}

A pesquisa envolveu 10.926 escolares. As análises mostraram que, quanto mais velhos os adolescentes, maior a insatisfação com o corpo. Quanto à dependência administrativa da escola, os alunos de instituições privadas são mais infelizes com a imagem (meninas: 33\% e meninos: 17,6\%) e tendem a ter mais atitudes para perder peso (meninas: 42,8\% e meninos: 28\%). O grau de instrução da mãe favorece as filhas a estarem mais insatisfeitas com o corpo (33,3\%) e os filhos a apresentarem mais atitudes para perder peso $(26,6 \%)$.

\section{Conclusão}

Condições sociodemográficas se relacionam à imagem corporal de adolescentes de maneira direta e indireta. Desvios negativos na imagem corporal podem resultar em distúrbios alimentares e sociais, prejudicando o desenvolvimento desses púberes. As consequências dessas ações se tornam um risco para a saúde e para o bem-estar psicológico, o que apoia a necessidade de iniciativas de programas voltados para questões relacionadas à imagem corporal no ambiente escolar.

Palavras-chave: Imagem corporal. Aparência física. Adolescente. Saúde pública.

\section{N TROD U C TIO N}

Body image may be set through the representation that the individuals make in their mind of their own body, based on their life sensations and experiences [1-4]. Human beings build their body image influenced by a number of factors of physical, psychological, environmental and cultural origin within the specific conditions of each person, such as: gender, age, ethnicity, socioeconomic and cultural condition [5-9].

The vision of body image occurs according to two dimensions: attitudinal and perceptive. The attitudinal dimension refers to the subject's behavior, emotions and cognitions about his/her body and appearance, and its components are: (dis)satisfaction, cognition and behavior $[3,10,11]$. The 
body image perceptual dimension refers to how the individual estimates the size and shape of his body $[1,3,10,12]$.

The formation of body image occurs parallel to development, that is, in the first years of the subject's life. As they grow up, young people build an image of themselves $[13,14]$. Thus, the period of puberty is an important phase for the formation of body image [15].

Individuals who are exposed to a negative body image may misjudge their body shape, being convinced that only other people are attractive; they obviously feel uncomfortable with their physical appearance, which will be associated with a low self-esteem, anxiety, depression and insecurity that affect social interactions and can become a factor in the performance of harmful behaviors to health [16].

Adolescents are susceptible to social pressures throughout their development. These young people form opinions about the perfect body, and the more the perfect body distances itself from the real body, the greater the possibility of conflict, affecting adolescents' self-esteem [17-18]. In search of the ideal body, some adolescents may adopt unhealthy behaviors, such as: excessive concern with weight control, reduced caloric intake, frequent restrictive diets; drugs and alcoholic beverages abuse; such behaviors may also feature obsessive, perfectionist, impulsive and affective instability traits, and in more severe cases can even lead to suicide $[16,18]$. These associations between negative body image and adoption of unhealthy behaviors, resulting in hampered physical and psychological health, is considered a serious public health concern $[17,18]$.

A recent study from Australia shows that $45.9 \%$ of girls have some concern about their bodies, while only $15.7 \%$ of boys have the same concern [19]. As to the prevalence of dissatisfaction with body image in developed countries it varies between $35 \%$ and $81 \%$ among female adolescents and between $16 \%$ and $55 \%$ among male adolescents $[20,21]$. In Brazil, the prevalence of body dissatisfaction is estimated around $70-75 \%$ in both genders [22].

The consequences of body dissatisfaction are closely associated with the etiology of eating disorders, including anorexia nervosa, bulimia nervosa and binge eating, especially in women. It has been calculated that 70 million people worldwide suffer from some type of eating disorder, with a fatality rate of $18 \%$ and 20\% [23]. A study carried out in the United States with a sample of 352 adolescents between 9 and 17 years of age, detected an average hospital stay for eating disorders of 18.4 days with an average cost per patient of $\$ 10,019.00[24]$.

Studies on body image occur mainly with female audiences, in urban areas, or with populations from specific regions [25-28]. Little is known about body image concerns and their relationship with sociodemographic conditions in a representative sample $[22,29]$. Thus, the objective of this study was to evaluate the relationship of associations between the dimensions of adolescents' body image and their sociodemographic conditions in Brazil using data from the Pesquisa Nacional de Saúde do Escolar (PeNSE, National School-based Health Survey).

\section{METHODS}

This is a cross-sectional study with secondary data from the Pesquisa Nacional de Saúde do Escolar (PeNSE, National School-based Health Survey), carried out in 2015. This survey was conducted by the Instituto Brasileiro de Geografia e Estatística (IBGE, Brazilian Institute of Geography and Statistics), in partnership with the Ministry of Health and the Ministry of Education [30].

Pesquisa Nacional de Saúde do Escolar investigated behavioral risk and health protection factors in a sample of students who attended primary and secondary schools, public and private, located in urban 
or rural areas throughout Brazil. The 2013 School Census was used to select the sample. The collection of research data took place during the months of April to September 2015, in parallel with the completion of the new School Census. Therefore, at the end of the collection, the results of the 2015 School Census were already available, which served to update the survey reference registration information [30].

Unlike previous versions, PeNSE 2015 was prepared with two samples, with Sample 1 being similar to previous versions with 9 th grade students, and Sample 2 consisting of students from $6^{\text {th }}$ grade to high school $3^{\text {rd }}$ grade. Sample 2 arises from the need for comparison with national and international indicators for the same audience, with representation for the age group from 13 to 17 years old. The sample was sized to estimate the parameters of interest in each of the five Major Regions of the country (North, Northeast, Southeast, South and Midwest) and, consequently, Brazil [30].

For the present study, we will use the PeNSE-2015 Sample 2 database; the survey involved students aged 13 to 17 years from public and private schools, located throughout Brazil, attending the $6^{\text {th }}$ to $9^{\text {th }}$ grade of elementary school and $1^{\text {st }}$ to $3^{\text {rd }}$ year of high school. Sample 2 estimates the parameters of interest in each of the Brazilian regions (North, Northeast, Midwest, Southeast and South) and in Brazil. The stratification of sample 2 grouped schools according to the region and, based on that, selected students through cluster sampling. All students from the selected classes present on that day were invited to answer the questionnaire, in both samples, as long as they were in agreement with the Informed Consent Form. This sample consisted of 380 schools, 652 classes and 16,608 students, which resulted in the completion of 10,926 valid questionnaires [30].

Data collection was performed using a self-administered questionnaire, using a smartphone, in which the structured questionnaire with thematic modules was inserted, and for the present study, the modules referring to sociodemographic characteristics and body image would be used. More details about the sampling process and the survey can be found in the PeNSE publication [30].

The main variables of interest in this study are those that assessed the attitudinal and perceptive dimensions of body image. The attitudinal dimension variables related to the satisfaction, cognition and behavior components were evaluated through the following questions: (a) For the feeling about their own body, which refers to the satisfaction component, we observed the answers obtained by the question "How do you feel about your body?" with the following possible answers: very satisfied, satisfied, indifferent, dissatisfied, very dissatisfied; the answers were subsequently grouped and re-categorized into indifferent, satisfied and dissatisfied; (b) The Degree of importance attributed to body image (cognition component) was obtained by the question "Do you consider your body image to be" ..., with the following possible answers: very important, important, little important and unimportant; the answers were subsequently grouped and re-categorized into important and unimportant; (c) To investigate the attitude towards one's own body (behavior component), we used the question "What are you doing in relation to your weight?" and, grouped the answers as do nothing, are you trying to lose weight, are you trying to keep your weight off and are you trying to gain weight.

The perceptual dimension was evaluated through the variable: self-perception of the nutritional status, obtained by the question "As for your body, do you consider yourself..." with the following possible answers: very thin, thin, normal, fat and very fat, being subsequently grouped and later categorized into thin, normal and fat.

The sociodemographic variables included gender (female and male), skin color (white, black, asian, mixed race, indigenous), age (grouped into: $\leq 13$ years, 14 and 15 years, $\geq 16$ years), regions (North, Northeast, Southeast, South and Midwest); living with parents (living with mother or father). Also mother's education (grouped into: no education, incomplete primary education, complete primary education, 
incomplete secondary education, complete secondary education, incomplete higher education, complete higher education, not informed) and school administrative status (public school and private school).

Initially, the relationship between the variables of the adolescents' body image dimensions (outcome) and the sociodemographic variables was assessed through the Pearson's chi-square test.

Association analyses using the univariate and multiple Poisson regression model, generating crude and adjusted prevalence ratios and their respective 95\% Confidence Intervals $(95 \% \mathrm{Cl})$ were conducted $\mathrm{A}$ regression model was performed for each body image variable (outcome), and for those that had three or more categories, indicator variables; the neutral response was considered as a reference category (for example, for the variable feeling about own body was considered the category "indifferent" as a reference). For the multiple models, all variables were included, and those significantly associated with the body image variables $(p<0.05)$ or those that changed the prevalence ratio by at least $10 \%$ were selected.

For data analysis, the Stata/SE version 11.2 program was used to obtain all estimates and to perform all analyses, considering the complex design of the sample (in cluster).

Pesquisa Nacional de Saúde do Escolar was approved by the Comissão Nacional de Ética em Pesquisa do Conselho Nacional de Saúde (CONEP, National Research Ethics Committee of the National Health Council), under registration No. 1.006,467, dated 03.30.2015. All students who participated in the survey agreed to their participation by signing the Free and Informed Consent Form - FICF. The research that generated this article was approved under the registry No. 4,099,758, dated 06.19.2020.

\section{R E S U L T S}

Data from 10,926 students attending the $6^{\text {th }}$ to $9^{\text {th }}$ grade elementary school (formerly $5^{\text {th }}$ to $8^{\text {th }}$ grades) and from $1^{\text {st }}$ to $3^{\text {rd }}$ year of high school were reviewed. The frequency proportions of responses for body image variables, in both genders, were higher for individuals who considered body image important. Among adolescents who had some image distortion, the highest prevalence was in females.

The variable feeling about one's own body (attitudinal dimension, component satisfaction), was related to the variables race/skin color, age, mother's education and administrative dependence in both genders. The degree of importance attributed to body image (cognition component) was associated with the age variable, in both genders. The attitude towards one's own body was significantly associated with all sociodemographic variables for males. In contrast, for females there was no statistical significance for the variables race/skin color and if there is the presence of the mother in the residence.

Table 1 shows the adjusted prevalence ratio for the attitudinal dimension - satisfaction component according to sociodemographic characteristics. Satisfaction with body image was significantly higher among male adolescents who declared themselves black (Prevalence Ratio (PR): 1.45; 95\% Confidence Intervals $(95 \% \mathrm{Cl}): 1.01-2.10 \%)$. Adolescents over 14 are the ones who are least satisfied with their bodies. When associated with maternal education, girls whose mothers have an incomplete higher education level are the ones who are most dissatisfied with their appearance (PR: 2.36; 95\% Cl: 1.04-5.36\%). On the other hand, students of both genders who studied in private schools showed lower satisfaction compared to students in public schools (Girls: PR: 0.76; 95\% Cl 0.59-0.99\%; Boys: PR: 0, 71; $95 \% \mathrm{Cl}$ $0.55-0.92 \%)$.

Table 2 shows the degree of importance attributed to body image. It was observed that girls from the Midwest region and those aged 13 years are the ones who consider their body image to be more significantly 
Table 1 - Poisson regression model results for the association between feeling about one's own body (attitudinal dimension, satisfaction component) and sociodemographic characteristics, according to gender. Brazil, 2015.

\begin{tabular}{|c|c|c|c|c|c|c|c|c|c|c|c|c|}
\hline \multirow{4}{*}{ Variables } & \multicolumn{12}{|c|}{ Feeling about own body } \\
\hline & \multicolumn{6}{|c|}{ Female } & \multicolumn{6}{|c|}{ Male } \\
\hline & \multicolumn{3}{|c|}{ Satisfied } & \multicolumn{3}{|c|}{ Unsatisfied } & \multicolumn{3}{|c|}{ Satisfied } & \multicolumn{3}{|c|}{ Unsatisfied } \\
\hline & Adjusted PR ${ }^{* *}$ & $95^{\circ}$ & $\mathrm{Cl}$ & Adjusted PR ${ }^{* *}$ & $95^{\circ}$ & $6 \mathrm{Cl}$ & Adjusted PR ${ }^{* *}$ & $95 \%$ & ${ }_{6}^{6} \mathrm{Cl}$ & Adjusted PR* & $95 \%$ & $\% \mathrm{Cl}$ \\
\hline \multicolumn{13}{|l|}{ Region } \\
\hline North & 1 & - & - & 1 & - & - & 1 & - & - & 1 & - & - \\
\hline Northeast & 1.04 & 0.74 & 1.46 & 0.94 & 0.63 & 1.39 & 1.02 & 0.76 & 1.37 & 1.41 & 0.98 & 2.01 \\
\hline Southeast & 0.86 & 0.61 & 1.21 & 1.02 & 0.69 & 1.5 & 1.01 & 0.75 & 1.37 & 1.27 & 0.89 & 1.83 \\
\hline South & 0.87 & 0.62 & 1.22 & 1.43 & 0.96 & 2.11 & 0.81 & 0.61 & 1.07 & 1.04 & 0.73 & 1.49 \\
\hline Midwest & 0.85 & 0.61 & 1.19 & 1.07 & 0.73 & 1.57 & 0.91 & 0.68 & 1.23 & 1.13 & 0.8 & 1.59 \\
\hline \multicolumn{13}{|l|}{ Race/color } \\
\hline White & 1 & - & - & 1 & - & - & 1 & - & - & 1 & - & - \\
\hline Black & 1.34 & 0.90 & 2.01 & 1.42 & 0.87 & 2.3 & $1.45^{*}$ & 1.01 & 2.1 & 1.06 & 0.7 & 1.6 \\
\hline Asian & 0.64 & 0.39 & 1.06 & 0.96 & 0.57 & 1.61 & 1.16 & 0.74 & 1.83 & 1.11 & 0.58 & 2.09 \\
\hline Mixed race & 1.05 & 0.82 & 1.33 & 1.13 & 0.87 & 1.47 & 1.16 & 0.93 & 1.44 & 1.01 & 0.72 & 1.4 \\
\hline Indigenous & 0.95 & 0.50 & 1.77 & 0.85 & 0.42 & 1.75 & 1.01 & 0.55 & 1.84 & 1.03 & 0.52 & 2.05 \\
\hline \multicolumn{13}{|l|}{ Age } \\
\hline under 13 years old & 1 & - & - & 1 & - & - & 1 & - & - & 1 & - & - \\
\hline 13 years & 0.97 & 0.68 & 1.39 & 1.27 & 0.94 & 1.71 & 1.01 & 0.75 & 1.34 & 1.01 & 0.65 & 1.55 \\
\hline 14 years & 0.96 & 0.71 & 1.29 & $1.54^{*}$ & 1.08 & 2.19 & 0.87 & 0.64 & 1.19 & 0.85 & 0.59 & 1.23 \\
\hline 15 years & 0.82 & 0.62 & 1.09 & $1.53^{*}$ & 1.13 & 2.06 & $0.59^{*}$ & 0.45 & 0.79 & 0.71 & 0.47 & 1.07 \\
\hline Over 16 years old & 0.9 & 0.68 & 1.19 & $1.64^{*}$ & 1.19 & 2.26 & $0.71^{*}$ & 0.54 & 0.95 & 0.97 & 0.68 & 1.39 \\
\hline \multicolumn{13}{|l|}{ Living with mother } \\
\hline No & 0.75 & 0.53 & 1.07 & 0.91 & 0.65 & 1.28 & 0.99 & 0.74 & 1.31 & 1.03 & 0.68 & 1.57 \\
\hline \multicolumn{13}{|l|}{ Living with father } \\
\hline No & 0.94 & 0.75 & 1.17 & 0.94 & 0.74 & 1.2 & 1.07 & 0.84 & 1.35 & 1.1 & 0.84 & 1.44 \\
\hline \multicolumn{13}{|l|}{ Maternal education } \\
\hline NS & 1 & - & - & 1 & - & - & 1 & - & - & 1 & - & - \\
\hline IES & 1.01 & 0.61 & 1.64 & 1.6 & 0.85 & 2.98 & 0.9 & 0.48 & 1.67 & 0.78 & 0.34 & 1.82 \\
\hline CES & 1.42 & 0.80 & 2.52 & 1.37 & 0.6 & 3.1 & 0.86 & 0.41 & 1.8 & 0.59 & 0.23 & 1.49 \\
\hline IHS & 0.79 & 0.45 & 1.41 & 1.17 & 0.54 & 2.5 & 1.15 & 0.55 & 2.39 & 1.53 & 0.6 & 3.9 \\
\hline $\mathrm{CHS}$ & 0.83 & 0.51 & 1.36 & 1.68 & 0.88 & 3.18 & 0.96 & 0.49 & 1.85 & 1.22 & 0.52 & 2.85 \\
\hline IHE & 1.01 & 0.55 & 1.87 & $2.36^{*}$ & 1.04 & 5.36 & 0.63 & 0.31 & 1.27 & 0.91 & 0.33 & 2.47 \\
\hline $\mathrm{CHE}$ & 0.99 & 0.59 & 1.69 & 1.84 & 0.92 & 3.67 & 0.81 & 0.41 & 1.6 & 1.07 & 0.44 & 2.58 \\
\hline DNI & 1.28 & 0.79 & 2.07 & 1.52 & 0.81 & 2.85 & 1.29 & 0.68 & 2.43 & 1.52 & 0.64 & 3.59 \\
\hline \multicolumn{13}{|c|}{ Administrative structure of the school } \\
\hline Public & 1 & - & - & 1 & - & - & 1 & - & - & 1 & - & - \\
\hline Private & $0.76^{*}$ & 0.59 & 0.99 & 1.32 & 0.98 & 1.79 & $0.71^{*}$ & 0.55 & 0.92 & 1.08 & 0.77 & 1.51 \\
\hline
\end{tabular}

Note: *Significant variables $(p<0.05)$ in Poisson regression mode; **Adjusted for socioeconomic variables associated with body image: region, race/color, age, living with mother, living with father, maternal education, administrative structure. CES: Complete Elementary School; CHE: Complete Higher Education; CHS: Complete High School; Cl: Confidence Interval; DNI: Did Not Inform; IES: Incomplete Elementary School; IHE: Incomplete Higher Education; IHS: Incomplete High School; NS: No Study; PR: Prevalence Reason.

important, but the adolescents who declared asian are the ones who believe that the concern with image is not so important (PR: $1.66 ; 95 \% \mathrm{Cl}: 1.06-2.61 \%$ ). Regarding maternal education, boys whose mothers have completed higher education are those who considered their physical appearance less important (PR: 1.57; 95\% Cl: 1.07-2.30\%).

The results on the attitudinal dimension, a behavioral component associated with sociodemographic characteristics (Tables 3 and 4), showed that female adolescents in the Southern region were the ones with 
Table 2 - Poisson regression model results for the association between the degree of importance attributed to body image (attitudinal dimension, cognition component) and sociodemographic characteristics according to gender. Brazil, 2015

\begin{tabular}{|c|c|c|c|c|c|c|}
\hline \multirow{4}{*}{ Variables } & \multicolumn{6}{|c|}{ Feeling about own body } \\
\hline & \multicolumn{3}{|c|}{ Female } & \multicolumn{3}{|c|}{ Male } \\
\hline & \multicolumn{3}{|c|}{ Unimportant } & \multicolumn{3}{|c|}{ Unimportant } \\
\hline & \multirow[t]{2}{*}{ Adjusted $\mathrm{PR}^{* *}$} & \multicolumn{2}{|c|}{$95 \% \mathrm{Cl}$} & \multirow[t]{2}{*}{ Adjusted PR ${ }^{* *}$} & \multicolumn{2}{|c|}{$95 \% \mathrm{Cl}$} \\
\hline \multicolumn{5}{|l|}{ Region } & & \\
\hline North & 1.00 & - & - & 1.00 & - & - \\
\hline Northeast & 0.81 & 0.62 & 1.04 & 0.99 & 0.79 & 1.24 \\
\hline Southeast & 0.82 & 0.64 & 1.04 & 0.98 & 0.78 & 1.23 \\
\hline South & 0.91 & 0.70 & 1.17 & 1.08 & 0.86 & 1.36 \\
\hline Midwest & $0.76^{*}$ & 0.60 & 0.96 & 0.99 & 0.79 & 1.25 \\
\hline \multicolumn{7}{|l|}{ Race/color } \\
\hline White & 1.00 & - & - & 1.00 & - & - \\
\hline Black & 0.96 & 0.72 & 1.29 & 1.03 & 0.82 & 1.28 \\
\hline Asian & $1.66^{*}$ & 1.06 & 2.61 & 0.99 & 0.65 & 1.50 \\
\hline Mixed race & 1.10 & 0.90 & 1.35 & 0.93 & 0.77 & 1.11 \\
\hline Indigenous & 1.07 & 0.68 & 1.69 & 1.26 & 0.88 & 1.82 \\
\hline \multicolumn{7}{|l|}{ Age } \\
\hline Under 13 years old & 1.00 & - & - & 1.00 & - & - \\
\hline 13 years & $0.72^{*}$ & 0.53 & 0.97 & 1.02 & 0.81 & 1.29 \\
\hline 14 years & 1.15 & 0.93 & 1.42 & 0.97 & 0.78 & 1.20 \\
\hline 15 years & 1.09 & 0.87 & 1.37 & 0.81 & 0.64 & 1.03 \\
\hline Over 16 years old & 0.85 & 0.65 & 1.11 & 0.81 & 0.64 & 1.03 \\
\hline \multicolumn{7}{|l|}{ Lives with the mother } \\
\hline No & 1.17 & 0.91 & 1.50 & 1.01 & 0.77 & 1.32 \\
\hline \multicolumn{7}{|l|}{ Lives with the father } \\
\hline No & 0.94 & 0.79 & 1.12 & 0.91 & 0.75 & 1.10 \\
\hline \multicolumn{7}{|l|}{ Maternal education } \\
\hline NS & 1.00 & - & - & 1.00 & - & - \\
\hline IES & 0.99 & 0.61 & 1.58 & 1.12 & 0.76 & 1.65 \\
\hline CES & 1.06 & 0.66 & 1.68 & 1.41 & 0.89 & 2.24 \\
\hline IHS & 1.32 & 0.76 & 2.28 & 1.35 & 0.83 & 2.20 \\
\hline $\mathrm{CHS}$ & 1.22 & 0.75 & 1.96 & 1.27 & 0.88 & 1.84 \\
\hline IHE & 1.37 & 0.78 & 2.38 & 1.47 & 0.91 & 2.36 \\
\hline $\mathrm{CHE}$ & 1.10 & 0.68 & 1.78 & $1.57^{*}$ & 1.07 & 2.30 \\
\hline DNI & 1.29 & 0.82 & 2.02 & $1.55^{*}$ & 1.06 & 2.29 \\
\hline \multicolumn{7}{|c|}{ Administrative dependence of the school } \\
\hline Public & 1.00 & - & - & 1.00 & - & - \\
\hline Private & 0.92 & 0.77 & 1.10 & 0.89 & 0.72 & 1.09 \\
\hline
\end{tabular}

Note: "Significant variables $(p<0.05)$ in Poisson regression model; **Adjusted for socioeconomic variables associated with body image: region, race/color, age, mother lives, father lives, maternal education, administrative dependence. CES: Complete Elementary School; CHE: Complete Higher Education; CHS: Complete High School; Cl: Confidence Interval; DNI: Did Not Inform; IES: Incomplete Elementary School; IHE: Incomplete Higher Education; IHS: Incomplete High School; NS: No Study; PR: Prevalence Reason.

the least attitudes of wanting to gain weight (PR: $0.71 ; 95 \% \mathrm{Cl}: 0.52-0.97$ ) (Table 3). Black boys, on the other hand, are the ones who least want to lose weight (PR: $0.71 ; 95 \% \mathrm{Cl}: 0.55-0.92 \%$ ) and indigenous boys are the ones who most want to gain a few kilos, compared to other teenagers (PR: 2.08 ; $95 \% \mathrm{Cl}: 1.17-3.72 \%$ ) (Table 4).

Male adolescents who live with their mothers have the lowest prevalence of attempting to lose weight (PR: 0.64; 95\% Cl: 0.49-0.84\%). When sociodemographic characteristics are associated with the attitudinal dimension - behavioral component, that is, related to attitudes towards their own bodies, 
Table 3 - Poisson regression model results for the association between attitude towards own body (attitudinal dimension, behavior component) and sociodemographic characteristics and female gender. Brazil, 2015.

\begin{tabular}{|c|c|c|c|c|c|c|c|c|c|}
\hline & \multicolumn{9}{|c|}{ Attitude towards own weight } \\
\hline & \multicolumn{9}{|c|}{ Female } \\
\hline & \multicolumn{3}{|c|}{ Lose weight } & \multicolumn{3}{|c|}{ Gain weight } & \multicolumn{3}{|c|}{ Keep weight } \\
\hline & Adjusted PR** & $95^{\circ}$ & $6 \mathrm{Cl}$ & Adjusted PR** & $95 \%$ & $6 \mathrm{Cl}$ & Adjusted PR** & $95 \%$ & $6 \mathrm{Cl}$ \\
\hline \multicolumn{10}{|l|}{ Region } \\
\hline North & 1.00 & - & - & 1.00 & - & - & 1.00 & - & - \\
\hline Northeast & 0.76 & 0.58 & 1.01 & 1.13 & 0.86 & 1.47 & 1.30 & 0.92 & 1.83 \\
\hline Southeast & 1.06 & 0.81 & 1.38 & 0.79 & 0.60 & 1.05 & 1.01 & 0.72 & 1.40 \\
\hline South & 1.32 & 1.00 & 1.75 & $0.71^{*}$ & 0.52 & 0.97 & 1.35 & 0.95 & 1.93 \\
\hline Midwest & 1.12 & 0.87 & 1.46 & 0.97 & 0.73 & 1.29 & 1.25 & 0.89 & 1.73 \\
\hline \multicolumn{10}{|l|}{ Race/color } \\
\hline White & 1.00 & - & - & 1.00 & - & - & 1.00 & - & - \\
\hline Black & 1.05 & 0.81 & 1.36 & 0.93 & 0.65 & 1.34 & 1.01 & 0.73 & 1.38 \\
\hline Asian & 1.20 & 0.83 & 1.74 & 1.04 & 0.66 & 1.64 & 1.02 & 0.70 & 1.48 \\
\hline Mixed race & 1.02 & 0.87 & 1.19 & 1.00 & 0.81 & 1.24 & 1.01 & 0.82 & 1.24 \\
\hline Indigenous & 1.43 & 0.97 & 2.10 & 0.98 & 0.58 & 1.67 & 0.99 & 0.54 & 1.82 \\
\hline \multicolumn{10}{|l|}{ Age } \\
\hline Under 13 years old & 1.00 & - & - & 1.00 & - & - & 1.00 & - & - \\
\hline 13 years old & $0.79^{*}$ & 0.63 & 0.98 & 0.95 & 0.71 & 1.26 & 0.87 & 0.69 & 1.10 \\
\hline 14 years old & 1.03 & 0.85 & 1.24 & 1.25 & 0.93 & 1.69 & 0.89 & 0.67 & 1.19 \\
\hline 15 years old & 1.15 & 0.93 & 1.44 & $1.45^{*}$ & 1.05 & 2.00 & 1.08 & 0.83 & 1.40 \\
\hline Over 16 years old & 1.04 & 0.83 & 1.29 & $1.40^{*}$ & 1.09 & 1.80 & 0.91 & 0.69 & 1.19 \\
\hline \multicolumn{10}{|l|}{ Lives with the mother } \\
\hline No & 1.13 & 0.89 & 1.43 & 0.95 & 0.68 & 1.34 & 1.01 & 0.69 & 1.48 \\
\hline \multicolumn{10}{|l|}{ Lives with the father } \\
\hline No & 0.94 & 0.81 & 1.08 & 1.06 & 0.85 & 1.31 & 0.95 & 0.81 & 1.11 \\
\hline \multicolumn{10}{|l|}{ Maternal education } \\
\hline NS & 1.00 & - & - & 1.00 & - & - & 1.00 & - & - \\
\hline IES & 1.35 & 0.90 & 2.02 & 1.16 & 0.73 & 1.83 & 1.42 & 0.98 & 2.05 \\
\hline CES & 1.20 & 0.74 & 1.95 & 0.97 & 0.55 & 1.69 & 1.45 & 0.89 & 2.36 \\
\hline IHS & 1.57 & 0.98 & 2.51 & 1.23 & 0.73 & 2.05 & 1.58 & 0.97 & 2.58 \\
\hline $\mathrm{CHS}$ & $1.62^{*}$ & 1.09 & 2.43 & 1.28 & 0.76 & 2.17 & 1.24 & 0.86 & 1.80 \\
\hline IHE & $1.75^{*}$ & 1.09 & 2.80 & 1.02 & 0.54 & 1.94 & 1.25 & 0.72 & 2.19 \\
\hline $\mathrm{CHE}$ & $1.88^{*}$ & 1.26 & 2.79 & 0.95 & 0.56 & 1.60 & 1.78 & 1.18 & 2.66 \\
\hline DNI & 1.02 & 0.71 & 1.47 & 0.90 & 0.53 & 1.51 & 0.96 & 0.66 & 1.38 \\
\hline \multicolumn{10}{|c|}{ Administrative structure of the school } \\
\hline Public & 1.00 & - & - & 1.00 & - & - & 1.00 & - & - \\
\hline Private & $1.67^{*}$ & 1.40 & 2.00 & 1.07 & 0.81 & 1.43 & $1.30^{*}$ & 1.01 & 1.68 \\
\hline
\end{tabular}

Note: "Significant variables $(\mathrm{p}<0.05)$ in Poisson regression model; ${ }^{* *}$ Adjusted for socioeconomic variables associated with body image: region, race/color, age, lives with the mother, lives with the father, maternal education, school administrative structure. CES: Complete Elementary School; CHE: Complete Higher Education; CHS: Complete High School; CI: Confidence Interval; DNI: Did Not Inform; IES: Incomplete Elementary School; IHE: Incomplete Higher Education; IHS: Incomplete High School; NS: No Study; PR: Prevalence Reason.

teenagers whose mothers have some level of education are the ones who try most to lose weight compared to teenagers whose mothers have no study. Regarding administrative dependence, students from private schools have a higher prevalence of behavioral attitudes towards their own body, both to lose weight (PR: 1.37; 95\%Cl: 1.13-1.66\%), and to gain weight (PR: 1.25 95\% Cl: 1.02-1.54\%) (Table 4).

In Table 5, the association of the perceptual dimension with sociodemographic characteristics is reported, in which girls from the Southern region (PR: $1.61 ; 95 \% \mathrm{Cl}: 1.24-2.10 \%$ ) and boys from 
Table 4 - Results of the Poisson regression model for the association between attitude towards own body (attitudinal dimension, behavior component (attitudinal dimension, behavior component) and sociodemographic characteristics and male gender. Brazil, 2015.

\begin{tabular}{|c|c|c|c|c|c|c|c|c|c|}
\hline \multirow{4}{*}{ Variables } & \multicolumn{9}{|c|}{ Attitude towards own weight } \\
\hline & \multicolumn{9}{|c|}{ Male } \\
\hline & \multicolumn{3}{|c|}{ Lose weight } & \multicolumn{3}{|c|}{ Gain weight } & \multicolumn{3}{|c|}{ Keep weight } \\
\hline & Adjusted PR** & $95^{\circ}$ & $\mathrm{Cl}$ & Adjusted PR** & & $6 \mathrm{Cl}$ & Adjusted PR** & $95 \%$ & $\mathrm{Cl}$ \\
\hline \multicolumn{10}{|l|}{ Region } \\
\hline North & 1.00 & - & - & 1.00 & - & - & 1.00 & - & - \\
\hline Northeast & 0.98 & 0.73 & 1.32 & 0.96 & 0.74 & 1.25 & Male & 0.61 & 1.18 \\
\hline Southeast & 0.99 & 0.75 & 1.30 & $0.73^{*}$ & 0.56 & 0.95 & 0.93 & 0.70 & 1.23 \\
\hline South & 1.14 & 0.88 & 1.48 & 0.77 & 0.59 & 1.01 & 0.95 & 0.71 & 1.27 \\
\hline Midwest & 1.14 & 0.87 & 1.51 & 0.78 & 0.59 & 1.02 & 1.01 & 0.76 & 1.36 \\
\hline \multicolumn{10}{|l|}{ Race/color } \\
\hline White & 1.00 & - & - & 1.00 & - & - & 1.00 & - & - \\
\hline Black & $0.71^{*}$ & 0.55 & 0.92 & 1.14 & 0.86 & 1.50 & 1.02 & 0.78 & 1.33 \\
\hline Asian & 1.11 & 0.74 & 1.65 & 1.22 & 0.79 & 1.88 & 1.36 & 0.85 & 2.17 \\
\hline Mixed race & 1.01 & 0.83 & 1.23 & 1.14 & 0.90 & 1.45 & 0.97 & 0.79 & 1.19 \\
\hline Indigenous & 1.45 & 0.95 & 2.22 & $2.08^{*}$ & 1.17 & 3.72 & 0.99 & 0.63 & 1.57 \\
\hline \multicolumn{10}{|l|}{ Age } \\
\hline Under 13 years old & 1.00 & - & - & 1.00 & - & - & 1.00 & - & - \\
\hline 13 years old & 0.78 & 0.58 & 1.05 & 1.21 & 0.90 & 1.62 & 0.82 & 0.62 & 1.07 \\
\hline 14 years old & $0.74^{*}$ & 0.60 & 0.91 & $1.44^{*}$ & 1.11 & 1.87 & 1.15 & 0.93 & 1.42 \\
\hline 15 years old & $0.58^{*}$ & 0.46 & 0.74 & $1.39^{*}$ & 1.05 & 1.82 & 0.86 & 0.66 & 1.11 \\
\hline Over 16 years old & $0.71^{*}$ & 0.58 & 0.87 & $1.61^{*}$ & 1.28 & 2.03 & 0.84 & 0.64 & 1.11 \\
\hline \multicolumn{10}{|l|}{ Lives with the mother } \\
\hline No & $0.64^{*}$ & 0.49 & 0.84 & 0.97 & 0.73 & 1.29 & 0.82 & 0.60 & 1.11 \\
\hline \multicolumn{10}{|l|}{ Lives with the father } \\
\hline No & 0.93 & 0.78 & 1.11 & 0.93 & 0.77 & 1.14 & 0.91 & 0.77 & 1.08 \\
\hline \multicolumn{10}{|l|}{ Maternal education } \\
\hline NS & 1.00 & - & - & 1.00 & - & - & 1.00 & - & - \\
\hline IES & $1.65^{*}$ & 1.11 & 2.45 & 1.48 & 0.96 & 2.29 & 1.44 & 0.93 & 2.22 \\
\hline CES & $1.93^{*}$ & 1.21 & 3.07 & $1.95^{*}$ & 1.16 & 3.28 & 1.48 & 0.86 & 2.56 \\
\hline IHS & $1.75^{*}$ & 1.09 & 2.80 & $2.08^{*}$ & 1.30 & 3.33 & 1.47 & 0.89 & 2.42 \\
\hline $\mathrm{CHS}$ & $1.65^{*}$ & 1.09 & 2.51 & $1.75^{*}$ & 1.14 & 2.68 & 1.53 & 0.99 & 2.35 \\
\hline IHE & $2.69^{*}$ & 1.65 & 4.40 & $2.95^{*}$ & 1.68 & 5.17 & 1.76 & 1.00 & 3.10 \\
\hline $\mathrm{CHE}$ & $1.92^{*}$ & 1.30 & 2.84 & $1.78^{*}$ & 1.19 & 2.68 & $1.68^{*}$ & 1.02 & 2.77 \\
\hline $\mathrm{DNI}$ & 1.31 & 0.89 & 1.92 & 1.30 & 0.87 & 1.96 & 1.35 & 0.87 & 2.09 \\
\hline \multicolumn{10}{|c|}{ Administrative structure of the school } \\
\hline Public & 1 & - & - & 1 & - & - & 1 & - & - \\
\hline Private & $1.37^{*}$ & 1.13 & 1.66 & $1.25^{*}$ & 1.02 & 1.54 & 0.97 & 0.78 & 1.21 \\
\hline
\end{tabular}

Note: "Significant variables $(\mathrm{p}<0.05)$ in Poisson regression model. ${ }^{* *}$ Adjusted for socioeconomic variables associated with body image: region, race/color, age, lives with the mother, lives with the father, maternal education, school administrative structure. CES: Complete Elementary School; CHE: Complete Higher Education; CHS: Complete High School; CI: Confidence Interval; DNI: Did Not Inform; IES: Incomplete Elementary School; IHE: Incomplete Higher Education; IHS: Incomplete High School; NS: No Study; PR: Prevalence Reason.

the Southeast region (PR: 1.40; 95\% Cl: 1.08-1.81) are the ones with the highest perception of feeling fat. The asian girls are the ones with the most distorted perception of normality. It was observed that age influences image distortion when compared to 13-year-old adolescents, in both genders (the older the more influence). Regarding the teaching place, students from private schools tend to see themselves as fatter than those from public schools (Girls: PR: 1.23; 95\% Cl: 1.01-1.49; Boys PR: 1.33; 95\% Cl: 1.03-1.72). 
Table 5 - Poisson regression model results for the association between self-perceived nutritional status (perceptual dimension) and sociodemographic characteristics and gender. Brazil, 2015.

\begin{tabular}{|c|c|c|c|c|c|c|c|c|c|c|c|c|}
\hline & \multicolumn{12}{|c|}{ Self-perception of body image } \\
\hline & \multicolumn{6}{|c|}{ Female } & \multicolumn{6}{|c|}{ Male } \\
\hline & \multicolumn{3}{|c|}{ Skinny } & \multicolumn{3}{|c|}{ Fat } & \multicolumn{3}{|c|}{ Skinny } & \multicolumn{3}{|c|}{ Fat } \\
\hline & Adjusted PR ${ }^{* *}$ & $95 \%$ & $\mathrm{Cl}$ & Adjusted PR ${ }^{* *}$ & $95^{\circ}$ & $6 \mathrm{Cl}$ & Adjusted PR** & $95 \%$ & ${ }_{6}^{6} \mathrm{Cl}$ & Adjusted PR** & $95 \%$ & $6 \mathrm{Cl}$ \\
\hline \multicolumn{13}{|l|}{ Region } \\
\hline North & 1.00 & - & - & 1.00 & - & - & 1.00 & - & - & 1.00 & - & - \\
\hline Northeast & 1.04 & 0.84 & 1.28 & 0.90 & 0.69 & 1.17 & $1.34^{*}$ & 1.06 & 1.69 & 1.24 & 0.94 & 1.64 \\
\hline Southeast & $1.28^{*}$ & 1.04 & 1.58 & $1.35^{*}$ & 1.04 & 1.74 & $1.23^{*}$ & 1.03 & 1.47 & $1.40^{*}$ & 1.08 & 1.81 \\
\hline South & 0.88 & 0.69 & 1.12 & $1.61^{*}$ & 1.24 & 2.10 & 1.10 & 0.88 & 1.38 & $1.39^{*}$ & 1.08 & 1.79 \\
\hline Midwest & 1.12 & 0.89 & 1.39 & $1.33^{*}$ & 1.03 & 1.71 & 0.98 & 0.77 & 1.24 & 1.25 & 0.98 & 1.61 \\
\hline \multicolumn{13}{|l|}{ Race/color } \\
\hline White & 1.00 & - & - & 1.00 & - & - & 1.00 & - & - & 1.00 & - & - \\
\hline Black & 0.95 & 0.74 & 1.24 & 1.24 & 0.95 & 1.61 & 0.98 & 0.79 & 1.22 & $0.68^{*}$ & 0.51 & 0.90 \\
\hline Asian & $1.45^{*}$ & 1.04 & 2.03 & $1.62 *$ & 1.15 & 2.30 & 0.95 & 0.62 & 1.46 & 0.75 & 0.43 & 1.28 \\
\hline Mixed race & 0.99 & 0.82 & 1.20 & 1.13 & 0.91 & 1.40 & 0.86 & 0.73 & 1.01 & 0.88 & 0.73 & 1.06 \\
\hline Indigenous & 0.76 & 0.48 & 1.19 & 1.17 & 0.74 & 1.85 & 1.01 & 0.66 & 1.53 & 0.96 & 0.64 & 1.45 \\
\hline \multicolumn{13}{|l|}{ Age } \\
\hline Under 13 years old & 1.00 & - & - & 1.00 & - & - & 1.00 & - & - & 1.00 & - & - \\
\hline 13 years old & $1.51^{*}$ & 1.22 & 1.87 & 1.04 & 0.81 & 1.33 & 1.24 & 0.99 & 1.57 & 0.81 & 0.60 & 1.08 \\
\hline 14 years old & $1.47^{*}$ & 1.18 & 1.82 & $1.27^{*}$ & 1.02 & 1.59 & $1.28^{*}$ & 1.04 & 1.58 & 0.87 & 0.66 & 1.14 \\
\hline 15 years old & $1.28^{*}$ & 1.04 & 1.58 & $1.43^{*}$ & 1.18 & 1.72 & $1.38^{*}$ & 1.13 & 1.67 & 0.78 & 0.59 & 1.04 \\
\hline Over 16 years old & $1.23^{*}$ & 1.02 & 1.48 & $1.50^{*}$ & 1.20 & 1.88 & $1.70^{*}$ & 1.35 & 2.13 & 0.90 & 0.69 & 1.16 \\
\hline \multicolumn{13}{|l|}{ Living with mother } \\
\hline No & 1.00 & 0.78 & 1.27 & 1.07 & 0.85 & 1.35 & 0.88 & 0.68 & 1.14 & 0.82 & 0.62 & 1.08 \\
\hline \multicolumn{13}{|l|}{ Living with father } \\
\hline No & 1.05 & 0.91 & 1.22 & 1.04 & 0.90 & 1.20 & 1.00 & 0.87 & 1.13 & 0.98 & 0.82 & 1.18 \\
\hline \multicolumn{13}{|l|}{ Maternal education } \\
\hline NS & 1.00 & - & - & 1.00 & - & - & 1.00 & - & - & 1.00 & - & - \\
\hline IES & 1.20 & 0.77 & 1.86 & 1.13 & 0.73 & 1.75 & 0.75 & 0.48 & 1.17 & 1.09 & 0.69 & 1.72 \\
\hline CES & 1.00 & 0.61 & 1.66 & 0.92 & 0.54 & 1.59 & 0.87 & 0.52 & 1.45 & 0.98 & 0.59 & 1.62 \\
\hline HIS & 1.16 & 0.74 & 1.82 & 1.19 & 0.71 & 2.00 & 0.85 & 0.52 & 1.39 & 1.11 & 0.69 & 1.77 \\
\hline $\mathrm{CHS}$ & 1.20 & 0.78 & 1.84 & 1.22 & 0.80 & 1.86 & 0.71 & 0.44 & 1.12 & 0.96 & 0.60 & 1.55 \\
\hline IHE & 1.16 & 0.72 & 1.85 & 1.43 & 0.82 & 2.48 & 1.04 & 0.65 & 1.68 & 1.13 & 0.65 & 1.96 \\
\hline $\mathrm{CHE}$ & 1.15 & 0.73 & 1.82 & 1.48 & 0.94 & 2.32 & 0.83 & 0.50 & 1.37 & 1.52 & 0.94 & 2.44 \\
\hline DNI & 1.09 & 0.71 & 1.68 & 1.10 & 0.74 & 1.65 & 0.79 & 0.51 & 1.20 & 0.91 & 0.59 & 1.41 \\
\hline \multicolumn{13}{|c|}{ Administrative structure of the school } \\
\hline Public & 1.00 & - & - & 1.00 & - & - & 1.00 & - & - & 1.00 & - & - \\
\hline Private & 1.10 & 0.90 & 1.34 & $1.23^{*}$ & 1.01 & 1.49 & 1.18 & 0.98 & 1.42 & $1.33^{*}$ & 1.03 & 1.72 \\
\hline
\end{tabular}

Note: *Significant variables $(\mathrm{p}<0.05)$ in Poisson regression model. ${ }^{* *}$ Adjusted for socioeconomic variables associated with body image: region, race/color, age, lives with the mother, lives with the father, maternal education, school administrative structure. CES: Complete Elementary School; CHE: Complete Higher Education; CHS: Complete High School; CI: Confidence Interval; DNI: Did Not Inform; IES: Incomplete Elementary School; IHE: Incomplete Higher Education; IHS: Incomplete High School; NS: No Study; PR: Prevalence Reason.

\section{I S C USSIO N}

Adolescence is a phase marked by transformations; young people are looking for the formation of their personality, which can trigger behaviors that neglect health care, making this population vulnerable [31]. In our study, students from private schools had greater image deviations, that is, they had lower satisfaction in both genders, with a greater perception of feeling fatter and, consequently, having more 
attitudes towards losing weight. In previous studies, it was noted that the administrative dependence of the school may indirectly reflect the individual's socioeconomic level. Thus, studies show that low-income students (going to public schools) wish to gain weight and higher-income students (going to private schools) want to lose weight $[32,33]$.

In relation to socioeconomic level, there is no methodological standard. It is possible to find several methods of analysis, like family income, parents' educational level and parents' occupation [34].

Mother's education can also be considered an indirect indicator of the adolescents' socioeconomic status in this study. The higher the mother's level of education, the greater the dissatisfaction of girls with their bodies. Regarding the attitudinal dimension, behavioral component, the greater the mother's education, the greater the desire to lose weight in both genders. In the literature, the surveys conducted show conflicting results; some surveys found that adolescents (aged 10 to 16), with mothers with less than nine years of education, were more likely to be dissatisfied with their bodies [35]. On the other hand, another study concluded that upper class subjects report significantly higher levels of food restriction, body dissatisfaction and body distortion [36]. Such divergences can be explained because the methods used in each study and the instruments used for evaluation, among others, are different.

Dissatisfaction with body image increases with age; this result is justified because children in the first stage of maturation do not suffer as much external influence, having only their parents as examples [37]. Over time, teenagers end up being persuaded by the standards that the media and the groups they belong to impose. Thus, agreeing with the current beauty standard can bring the illusion of status and the possibility of being socially accepted [38].

The present study also found an association between the presence of adolescents living with their parents and the dimensions of body image. Boys who live with their mothers show behavioral attitudes towards weight loss. Previous studies corroborate our findings, showing that adolescents had the perception that their parents and friends would like them to be thinner, since in the early years of adolescence, parents exert a great influence on the appearance and style of their children [38]. These parents, through unhealthy behaviors, such as: expressing dissatisfaction with the child's weight; carry out restrictive diets; being concerned about their own weight, may generate opportunities for their children to imitate these values, attitudes and behaviors [33,38].

Regarding the age group of adolescents, it was found that, in both genders, adolescents aged 15 years and over are dissatisfied with their bodies, as they have the self-perception of feeling thinner and, consequently, practice attitudes to gain weight. Boys, in addition to wanting to gain weight, also have attitudes to lose weight. It should be noted that sociocultural patterns interfere differently for boys and girls; boys are encouraged to play sports to gain muscle, while girls are encouraged to participate in activities to control or lose weight. In this way, boys can associate weight gain with muscle gain [39].

Body image in adolescents has been associated, in some studies, with sexual maturation $[38,39]$. Adolescents, at the beginning of their maturation, have a childlike body and mentality. Therefore, a higher prevalence of satisfaction with the image was observed. At the end of maturation, these young people have characteristics that are very similar to adults', and they may have already incorporated the beauty ideals of our culture $[38,39]$.

When associated with image dimensions, with adolescent skin color, it was found in the present study that black boys are more satisfied with their physical appearance, thus not having image deviations in the attitudinal dimension nor in the perceptual dimension. A study on the subject presents similar results, noting that African-American boys have a weaker association of body dissatisfaction with unhealthy weight control behaviors than for white, Asian or mixed race boys [40]. 
According to the location of the young people, girls from the Southern region are the ones that interfered in the perceptual dimension, while boys from the Southeast region showed change in perception because they considered themselves fatter. Research has shown that the prevalence of body image dissatisfaction is similar across geographic locations. It is believed that there are more negative complications in body image in adolescents living in more densely populated areas and with more access to information, who could be more exposed to social pressure from the slimness culture [41]. Regions with lower population density have low commercial entertainments available (malls, cinemas, gyms, among others), collaborates with the low influence on the enhancement of physical appearance, protecting them from messages conveyed about the ideal female and male bodies models [42]. A study conducted with 607 children showed similar results to this study, in which there is a higher rate of image dissatisfaction among young people in urban areas [43].

Distortions in all dimensional spheres of body image can result in eating and social disorders impairing adolescents' development. With the increasing rate of obesity among this audience, public policies tend to focus only on preventing overweight/obesity and weight loss. However, interventions that address the dimensions of body image are of paramount importance [44]. Research in the mental health area shows that there is a lack of care and prevention programs for children and youth mental health problems, and that there is also a lack of policies that map and train professionals in this area to identify future problems related, for example, with body image distortions [44].

Schools have been assuming a growing importance in promoting health and preventing illnesses among children and adolescents, mainly because most of these individuals spend most of their time in a school setting. The place of education should be able to prepare students to be able to take care of their own health, adopting healthy behaviors in all phases of their lives $[45,46]$.

The consequences of the formation of negative body image making it a risk to health and psychological well-being strongly support the initiative of programs aimed at issues related to body image in the school setting. A British parliamentary inquiry recommended that evidence-based body image classes be mandatory in primary and secondary school curricula $[45,46]$.

A US survey suggests seven steps that encourage education promotion aimed at enhancing a positive body image, such as: Health promotion should minimize the potential discrimination in relation to the body; When dealing with obesity specifically addressing the positive or adverse effects of body image It is necessary to encourage increased physical activity to improve body satisfaction; Enable health professionals to understand the topic and know how to apply the knowledge about promotion of body satisfaction in practice; These professionals should provide information to parents in order to foster positive behaviors in the family setting. Anti-bullying policies must be implemented in schools and the school curriculum must include content aimed at promoting positive body image to prevent potential risks for the future of these adolescents $[45,46]$.

Thus, one of the limitations of the study is its cross-sectional nature, which implies that the temporal sequence of events was not considered, making it impossible to determine causality. Longitudinal studies would provide a deeper understanding of the causal pathways involved. In addition, the use of a selfadministered questionnaire may present response biases, due to the student's embarrassment when answering; thus, some responses may underestimate or overestimate the indicators assessed. Another limitation is the adherence to the answers, for example, $25.31 \%$ of the students were unable to report the level of maternal education, which can impact the results.

Although PeNSE uses a self-administered questionnaire, the scope of an epidemiological study, representative of the elementary and secondary school population, allows us to assess multiple factors that 
interfere with body image and its dimensions, in an unprecedented way. Those factors provide important evidence aimed at this audience, which can support policies and campaigns that positively affect this population health associated with sociodemographic characteristics.

\section{CONCLUSION}

Based on this study's results it was possible to observe an association between body dimensions and sociodemographic variables in adolescents. Body image can be directly or indirectly influenced by sociodemographic factors, such as the place where you live, administrative status of the school, presence of parents at home, maternal education, age, ethnicity, among others.

In addition, disorders in body dimensions can result in eating and social disorders, impairing the development of adolescents, further emphasizing the need to provide public policies for the implementation of different prevention actions, with possibilities for discussions and reflections, especially in the psychological field. The school plays an important role in promoting these young people's health, preparing adolescents for a promising future, avoiding physical and emotional deterioration in relation to their physical appearance through educational initiatives aimed at raising awareness about positive body image.

\section{CONTRIBUTORS}

LS BATISTA participated in the conception, planning, study, analysis and interpretation of data, preparing the first version. HVB GONÇALVES participated in the study, analysis, data interpretation and review of the final version of the manuscript. DH BANDONI participated in the study planning, data analysis and interpretation, critical content review and approved the final version of the manuscript.

\section{REFERENCES}

1. Oliveira, N. Body image and its relationship with food consumption according to the NOVA classification: results from the Pró-Saúde Study, 2018 [dissertation]. Rio de Janeiro: Universidade Estadual do Rio de Janeiro; 2018.

2. Scatolin, HG. The body image: the constructive energies of the psyche. Rev Psic. 2012;21:115-20.

3. Tavares MC, Campana ANNB, Tavares Filho RF, Campana MB. Perceptual assessment of body image: history, reconception and perspectives for Brazil. Psicol Est. 2010;15(3):509-18.

4. Castro IRRD, Levy RB, Cardoso LDO, Passos MDD, Sardinha LMV, Tavares LF, et al. Body image, nutritional status and weight behavior among Brazilian adolescents. Ciênc Saúde Coletiva. 2010;15:3099-108.

5. Veras ALL. Development and construction of body image today: a cognitive-behavioral look. Rev Bras Ter Cogn. 2010;6(2):94-117.

6. Campos MTD, Avoglia HRC, Custodio EM. Body image of individuals with non-congenital paraplegia: an exploratory study. Psicol Inf. 2007;11(11):27-55.

7. Barros DD. Body image: the discovery of oneself. Hist Cienc Saude-Manguinhos. 2005;12(2):547-54.

8. Turtelli LS, Tavares MCGCF, Duarte E. Paths of body image research in its relationship with movement. Rev Bras Ciênc Esp. 2002;24(01):151-66.

9. Grogan S. Body Image: understanding body dissatisfaction in men, women, and children. London: Routledge, 1999.

10. Neves NA, Morgado FFDR, Tavares MCGCF. Body image assessment: essential notes for good research practice. Psic Content Res. 2015;31(3):375-80.

11. Thompson JK. The (mis)measurement of body image: ten strategies to improve assessment for applied and research purposes. Body Image. 2004;1:7-14. 
12. McCabe M, Ricciardelli LA, Sitaram G, Mikhail K. Accuracy of body size estimation: role of biopsychosocial variables. Body Image. 2006;3:163-71.

13. Pop C. Self-esteem and body image perception in a sample of university students. Eurasian J Edu Res. 2016;16:31-44.

14. Anaruma, SM. Meeting with the body: a psychological intervention program for the care of overweight people [dissertation]. Campinas: Universidade Estadual de Campinas; 1995.

15. Abou Abbas O; Albuhairan, F. Predictors of adolescents' mental health problems in Saudi Arabia: findings from the Jeeluna ${ }^{\circledR}$ national study. Child Adol Psyc Mental Health. 2017;11(52).

16. Cruz-Bojórquez RM, Coop-Gamas FY, Cárdenas-García S, Ávila-Escalante ML. Perception of body image in Maya adolescents and its relationship with body dissatisfaction and nutritional status. J Nutr Food Sci. 2019;9:756.

17. Valença CN, Germano RM. Perception of self-image and body satisfaction in adolescents: perspective of comprehensive nursing care. RENE. 2009;10(4):173-80.

18. Morgan CM, Vecchiatti IR, Negrao AB. Etiology of eating disorders: biological, psychological and socio-cultural aspects. Rev Bras Psiquiatr. 2002;24(3):18-23.

19. Tiller E, Fildes J, Hall S, Hicking V, Groenlândia N, Liyanarachchi D, et al. Youth Survey Report 2020. Sydney, NSW: Mission Australia; 2020.

20. Ribeiro-Silva RC, Fiaccone RL, Conceição-Machado MEP, Ruiz AS, Barreto ML, Santana MLP. Body Image Dissatisfaction and dietary patterns according to nutritional status in adolescents. J Pediatric. 2018:94(2):155-61.

21. Lawler M, Nixon E. Body dissatisfaction among adolescent boys and girls: the effects of body mass, peer appearance culture and internalization of appearance ideals. J Youth Adolesc. 2011;40:59-71.

22. Laus MF, Miranda VP, Almeida SS, Braga Costa TM, Ferreira ME. Geographic location, sex and nutritional status play an important role in body image concerns among Brazilian adolescents. J Health Psychol. 2013;14(3):508-21.

23. Santos MFC, Vieira ZN, Souza EA, Longo CS. Eating disorders in femaleadolescents: a review study. Psicologia. 2019.

24. Robergeau K, Joseph J, Silber TJ. Hospitalization of children and adolescents for eating disorders in the state of New York. J Adol Health. 2006;39(6):806-10.

25. Fortes LS, Filgueiras JF, Oliveira FC, Almeida SS, Ferreira MEC. Etiological model of risk behaviors for eating disorders in Brazilian female adolescents. Cad Saúde Pública. 2016;32(4).

26. Contreras AML, Morán KJ, Frez HS, Lagos OC, Marín FMP, Pinto BMA, et al. Weight management conducts in adolescent dieting women and their relationship with body dissatisfaction and obsession with delegation. Rev Chil Pediatr. 2015;86(2):97-102.

27. Rentz-Fernandes AR, Silveira-Viana M, Liz CM, Andrade A. Self-esteem, body image and depression of adolescents in different nutritional states. Rev Salud Pública. 2017;19(1):6-72.

28. Felden ÉPG, Claumann GS, Sacomori C, Daronco LSE, Cardoso FL, Pelegrini A. Sociodemographic factors and body image in high school adolescents. Ciênc Saúde Coletiva. 2015;20(11):3329-37.

29. Fidelix Y, Silva D, Pelegrini A, Silva A, Petroski E. Body image dissatisfaction among adolescents from a small town: association with gender, age, and area of residence. Ver Bras Cineantropom Desempenho Hum. 2011;13(3):202-7.

30. Instituto Brasileiro de Geografia e Estatística. Pesquisa Nacional de Saúde do Escolar. Rio de Janeiro: Instituto; 2015.

31. Fonseca FF, Sena RKR, Santos RLA, Dias OV, Costa SM. Vulnerabilities in childhood and adolescence and Brazilian public intervention policies. Rev Paul Pediatr. 2013;31(2):258-64.

32. Fernandes AER. Assessment of body image, lifestyle and eating habits in children and adolescents from public and private schools in Belo Horizonte [dissertation]. Belo Horizonte: UFMG; 2007.

33. Zaimin W, Nuala MB, Justin AK, Andrew PH. Influences of ethnicity and socioeconomic status on the body dissatisfaction and eating behaviour of Australian children and adolescents. Eating Behaviors. 2005;6(Issue 1):23-33.

34. Instituto Nacional de Estudos e Pesquisas Anísio Teixeira (Brasil). Saeb 2019: indicador de nível socioeconômico do Saeb 2019: nota técnica. Brasília: INEP, 2021.

35. Marques FA, Legal EJ, Höfelmann DA. Body dissatisfaction and common mental disorders in adolescents. Rev Paul Pediatr. 2012;30(4):553-61.

36. Ogden J, Thomas D. The role of familial values in understanding the impact of social class on weight concern. Int J Eat Disord. 1999;25(3):273-9. 
37. Neha R, Lynn R, Anthony W. What influences urban Indian secondary school students' food consumption? A qualitative study. Appetite, 2016;105:790-7.

38. Pinheiro AP, Giugliane ERJ. Who are children with adequade weight who feel fat? J Pediatria. 2006;82(3):232-5.

39. Aerts D, Madeira RR, Zart VB. Body image of school adolescents in Gravataí-RS. Epidemiol Serv Saúde. 2010;19(3): 283-91.

40. Bucchianeri MM, Fernandes N, Loth K, Hannan PJ, Eisenberg ME, Neumark-Sztainer D. Body dissatisfaction: do associations with eating disorders and psychological well-being differ between race/ethnicity in adolescent girls and boys? Cultur Divers Ethnic Minor Psychol. 2016;22(1):137-46.

41. Petroski EL, Pelegrini A, Glaner MF. Body dissatisfaction in rural and urban adolescents. Motri. 2009;5(4):13-25.

42. Miranda VPN, Conti MA, Bastos RR, Laus MF, Almeida SS, Ferreira MEC. Body image of adolescents in rural cities. Ciênc Saúde Coletiva. 2014;19(06):1791-801.

43. Triches RM, Giugliani ERJ. Body dissatisfaction in two municipalities in southern Brazil. Rev Nutr. 2007;20(2):119-28.

44. Voelker DK, Reel JJ, Greenleaf C. Weight status and body image perception in adolescents: current perspectives. Adolesc Health Med Ther. 2015;6:149-58.

45. Bucchianeri M, Neumark-Sztainer D. Body dissatisfaction: an overlooked public health concern. J Public Mental Health, 2014:13(2):64-9.

46. Zali Y, Phillippa CD, Lina AR, Emma H. What works in secondary schools? A systematic review of classroom-based body image programs. Body Image. 2013:10(3):271-81. 